\title{
SYNTHESIS OF AN LMI-BASED FUZZY CONTROL SYSTEM WITH GUARANTEED COST PERFORMANCE: A PIECEWISE LYAPUNOV APPROACH
}

\author{
Natache S. D. Arrifano* \\ natachea@sel.eesc.usp.br
}

\author{
Vilma A. Oliveira* \\ vilmaodsel.eesc.usp.br
}

\author{
Lúcia V. Cossi ${ }^{\dagger}$ \\ cossi@ufpb.br \\ *Departamento de Engenharia Elétrica, Universidade de São Paulo \\ Av. Trabalhador São Carlense, 400 \\ CEP 13566-590, São Carlos, SP, BRASIL \\ ${ }^{\dagger}$ Departamento de Matemática, Universidade Federal da Paraíba \\ Cx. Postal 5080 \\ CEP 58051-970, João Pessoa, PB, BRASIL
}

\begin{abstract}
A new stability analysis and design of a fuzzy switching control based on uncertain Takagi-Sugeno fuzzy systems are proposed. The fuzzy system adopted is composed by a family of local linear uncertain systems with aggregation. The control design proposed uses local state feedback gains obtained from an optimization problem with guaranteed cost performance formulated in the context of linear matrix inequalities and a fuzzy switching scheme built from local Lyapunov functions. The global stability is guaranteed by considering a class of piecewise quadratic Lyapunov functions. Examples are given to illustrate the applicability of the proposed approach.
\end{abstract}

KEYWORDS: Switching fuzzy control, Guaranteed cost fuzzy control, Uncertain Takagi-Sugeno fuzzy systems, Piecewise quadratic Lyapunov functions.

\footnotetext{
Artigo submetido em $28 / 11 / 2002$

1a. Revisão em 26/06/2003

2a. Revisão em 18/03/2004

3a. Revisão em 29/05/2006

Aceito sob recomendação do Editor Associado

Prof. Cairo Lucio Nascimento Jr
}

\section{RESUMO}

Neste trabalho, uma nova análise de estabilidade e projeto de controle fuzzy chaveado baseado em sistemas fuzzy TakagiSugeno com incertezas são propostos. O sistema fuzzy adotado é composto por uma família de sistemas lineares incertos locais com agregação fuzzy. O projeto de controle proposto utiliza ganhos de realimentação de estado locais obtidos da solução de um problema de otimização com desempenho de custo garantido formulado em termos de desigualdades matriciais lineares e um esquema de chaveamento fuzzy baseado em funções de Lyapunov, que são usadas quando a trajetória do estado do sistema está na fronteira de subespaços definidos do espaço de estado. A estabilidade global é garantida considerando uma classe de funções de Lyapunov quadráticas por partes. Exemplos ilustram a aplicação da abordagem proposta.

PALAVRAS-CHAVE: Controle fuzzy chaveado, Controle fuzzy de custo garantido, Sistemas fuzzy Takagi-Sugeno com incertezas, Funções de Lyapunov quadráticas por partes. 


\section{INTRODUCTION}

Takagi-Sugeno (TS) fuzzy-model-based control has been successful used to control nonlinear systems in several applications (Tanaka et al., 1999; Feng et al., 1997; Wang et al., 1996). Most of the techniques of robust control have been used in the TS fuzzy-model-based control due to the fact that the TS fuzzy systems can be interpreted as differential inclusions (Jadbabaie et al., 1998a; Cao et al., 1997; Tanaka et al., 1996). However, when treating uncertain nonlinear systems, we need to distinguish nonlinearity from uncertainty, otherwise, the results obtained are in general conservatives. Several approaches have appeared to the robust stabilization of uncertain nonlinear systems (Lee et al., 2001; Cao et al., 2001; Teixeira and Żak, 1999; Tanaka et al., 1996). In the framework of TS fuzzy systems, parametric uncertainty can be represented by norm-bounded or polytopic uncertain sets. Different robust control solutions for the TS fuzzy system with norm-bounded and polytopic uncertainty representations can be found in Tanaka et al. (1996) and Lee et al. (2001), and Cao et al. (2001), respectively.

Stability is one of the most important issues when analyzing control systems. Most of the methods of fuzzy-model-based control yields stability analysis and design procedures by means of the parallel distributed compensation (PDC) using a common quadratic Lyapunov function (Teixeira et al., 2000; Tanaka et al., 1998; Tanaka et al., 1997). This approach requires a common positive definite matrix that is a solution of all the Lyapunov inequalities built from the local linear systems of the global feedback TS fuzzy system, which are usually formulated in terms of linear matrix inequalities (LMI's) in both the state feedback gain and Lyapunov matrix. However, when applied to uncertain nonlinear systems, this approach may not provide feasible results because it is not possible to find a common positive definite Lyapunov matrix as a solution of several Lyapunov inequalities. To remove this deficiency, recently, attractive stability results for the TS fuzzy-model-based control using piecewise quadratic Lyapunov functions appeared (Zhang et al., 2001; Johansson et al., 1998). These results explore the gain-scheduled nature of the fuzzy controllers and have found application in the stability analysis of systems whose dynamics depends on the subspace of the state space their trajectory is.

This paper presents a fuzzy switching controller for uncertain nonlinear systems which are represented by a class of TS fuzzy systems with uncertainties. The controller proposed uses local guaranteed cost control laws and a switching scheme based on local quadratic Lyapunov functions when the state is on the boundary of defined subspaces of the state space. A sufficient condition for the stability of the uncertain nonlinear system with state feedback is given in terms of a piecewise quadratic Lyapunov function. This approach pro- duces less conservative results than those obtained with the fuzzy blending controller for TS fuzzy systems with uncertainties. In addition, this approach may be applied to control highly nonlinear systems, where available robust control techniques are not successful.

The remainder of the paper is organized as follows. In Section 2, the fuzzy system modeling for a class of uncertain nonlinear systems, the fuzzy switching control and the guaranteed cost control design are presented. The stability analysis of the feedback fuzzy system is the subject of the Section 3. In Section 4, simulation results are presented to illustrate the effectiveness of the proposed approach. Finally, the paper concludes with brief remarks in Section 5.

\section{FUZZY SYSTEM MODELING AND CON- TROL}

We consider a class of uncertain nonlinear dynamic systems which are described by the differential inclusion

$$
\dot{x} \in C o\left\{f_{k}(x)+g_{k}(x) u\right\}, x(0)=x_{0},
$$

where $x$ is the system state vector, $u$ is the input vector, $C o$ denotes the convex hull, $f_{k}(\cdot), g_{k}(\cdot)$ are smooth nonlinear functions which define the called vertex systems, $f_{k}(0)=0$, $g_{k}(0)=0$, and $v$ is the number of vertexes, $k=1,2, \ldots, v$, with $f_{k}: \mathbb{R}^{n} \rightarrow \mathbb{R}^{n}$ and $g_{k}: \mathbb{R}^{n} \rightarrow \mathcal{F}\left(\mathbb{R}^{m}, \mathbb{R}^{n}\right)$ for $\mathcal{F}\left(\mathbb{R}^{m}, \mathbb{R}^{n}\right)=\left\{h: \mathcal{D}(h) \rightarrow \mathbb{R}^{n}: \mathcal{D}(h) \subseteq \mathbb{R}^{m}\right.$.

Considering an uncertain parameter vector $p \in \mathbb{R}^{s}$, a polytopic representation of (1) has the form

$$
\dot{x}=\sum_{k=1}^{v} \eta_{k}(p)\left(f_{k}(x)+g_{k}(x) u\right),
$$

where $\eta_{k}: \mathbb{R}^{s} \rightarrow \mathbb{R}$ with $\eta_{k}(p) \geq 0, k=1,2, \ldots, v$, $\sum_{k=1}^{v} \eta_{k}(p)=1$.

\subsection{Fuzzy system modeling}

The TS fuzzy system is described by fuzzy IF-THEN rules representing local input-output relations of the nonlinear system (Takagi and Sugeno, 1985). The basic idea of this approach is decompose the input space into many subspaces, approximating the nonlinear system by a blending of the local linear systems associated to each subspace. In fact, it is proved that the TS fuzzy systems are universal approximators (Tanaka and Wang, 2001). In order to consider uncertainties in the TS fuzzy system, we use a fuzzy system built from local uncertain linear systems whose the $i$ th inference 
rule is given by

Rule $i$ :

$$
\begin{aligned}
& \text { If } x_{1} \text { is } F_{1}^{i} \text { and } x_{2} \text { is } F_{2}^{i} \text { and } \ldots x_{n} \text { is } F_{n}^{i} \\
& \text { Then } \dot{x}=A_{i}(p) x+B_{i}(p) u \\
& i=1,2, \ldots, r,
\end{aligned}
$$

where $F_{j}^{i}, j=1,2, \ldots, n$ are fuzzy sets, $r$ is the number of inference rules, and matrices $A_{i}(p) \in \mathcal{M}\left(\mathbb{R}^{n}, \mathbb{R}^{n}\right)$ and $\left.B_{i}(p)\right) \in \mathcal{M}\left(\mathbb{R}^{n}, \mathbb{R}^{m}\right)$ have a polytopic representation, that is, $A_{i}(p)=\sum_{k=1}^{v} \eta_{k}(p) A_{i k}$ and $B_{i}(p)=\sum_{k=1}^{v} \eta_{k}(p) B_{i k}$, for $\eta_{k}(p) \geq 0, k=1,2, \ldots, v, \sum_{k=1}^{v} \eta_{k}(p)=1$. Matrices $A_{i}(p)$ and $B_{i}(p), i=1,2, \ldots, r$ can be obtained from (2) using the linearization formula proposed by Teixeira and Żak (1999), which yields a good linear approximation of nonlinear systems in the vicinity of an operating point even if it is not an equilibrium point. The Teixeira \& Żak linearization formula used in this paper is presented in Appendix A.

Given the pair $(x, u)$, the overall fuzzy system with uncertainties is inferred as a weighted average of all local uncertain linear approximations $\left(A_{i}(p), B_{i}(p)\right), i=1,2, \ldots, r$ of (3), which is given by

$$
\dot{x}=\sum_{i=1}^{r} \alpha_{i}(x)\left(A_{i}(p) x+B_{i}(p) u\right)
$$

where

$$
\alpha_{i}(x)=\frac{\prod_{j=1}^{n} F_{j}^{i}\left(x_{j}\right)}{\sum_{i=1}^{r} \prod_{j=1}^{n} F_{j}^{i}\left(x_{j}\right)}
$$

denotes the normalized membership function, with $F_{j}^{i}\left(x_{j}\right) \in$ $[0,1]$ the grade of membership of $x_{j}, j=1,2, \ldots, n$, in the fuzzy set $F_{j}^{i}$. Considering the fact that in (5) $F_{j}^{i}\left(x_{j}\right) \geq 0$, $i=1,2, \ldots, r$ and $j=1,2, \ldots, n$, we have $\alpha_{i}(x) \geq 0$ and $\sum_{i=1}^{r} \alpha_{i}(x)=1, \forall t \geq 0$.

\subsection{Fuzzy switching control}

In this section we propose a switching scheme so that local controllers are switched according to the subspace that the state vector $x$ enters. For this purpose, let $S_{i}^{\circ}$ denote the $i$ th subspace in the state space

$$
S_{i}^{\circ}:=\left\{x \mid \alpha_{i}(x)>\alpha_{\ell}(x) ; i \neq \ell ; i, \ell=1,2, \ldots, r\right\},
$$

where the superscript ${ }^{\circ}$ in $S_{i}$ denotes an open subspace, let $\partial S_{i \ell}$ denote the transition subspace in the state space

$$
\partial S_{i \ell}:=\left\{x \mid \alpha_{i}(x)=\alpha_{\ell}(x) ; i \neq \ell ; i, \ell=1,2, \ldots, r\right\},
$$

and let $S_{i}=S_{i}^{o} \bigcup \partial S_{i \ell}$. Using (6) and (7), we define the switching scheme for each rule $i$

Rule $i$ :

$$
\begin{array}{ll}
\text { If } & x \in S_{i}^{\circ} \\
\text { Then } & \beta_{i}(x)=1 \\
\text { If } & x \in \partial S_{i \ell} \text { and } V_{i}(x) \leq V_{\ell}(x) \\
\text { Then } & \beta_{i}(x)=1 \text { and } \beta_{\ell}(x)=0 \\
& i \neq \ell, i, \ell=1,2, \ldots, r,
\end{array}
$$

where $V_{i}(x)=x^{T} P_{i} x$ is a local quadratic Lyapunov function, with $P_{i}=P_{i}^{T}, P_{i}>0$ and $\beta_{i}(x) \in\{0,1\}$ a crisp function which changes as $x$ leaves subspace $S_{i}^{\circ}$ following the membership function changes. Thus, $\beta_{i}(x)=1$ only when $x \in S_{i}=S_{i}^{\circ} \cup \partial S_{i \ell}$ and $\beta_{i}(x)=0$, otherwise. Additionally, $\sum_{i=1}^{r} \beta_{i}(x)=1, \forall t \geq 0$.

Adopting (8) and following the idea of the PDC scheme, we propose the fuzzy switching control as

$$
u=-\sum_{i=1}^{r} \beta_{i}(x) K_{i} x,
$$

where $K_{i} \in \mathcal{M}\left(\mathbb{R}^{m}, \mathbb{R}^{n}\right), i=1,2, \ldots, r$ are the state feedback gains to be designed for rule $i$. In order to obtain the state feedback fuzzy system, we substitute (9) in (4), which gives

$$
\begin{aligned}
\dot{x}= & \sum_{i=1}^{r} \alpha_{i}(x)\left[A_{i}(p)-\left(\sum_{j=1}^{r} \beta_{j}(x) B_{i}(p) K_{j}\right)\right] x \\
& \sum_{i=1}^{r} \sum_{j=1}^{r} \alpha_{i}(x) \beta_{j}(x)\left(A_{i}(p)-B_{i}(p) K_{j}\right) x
\end{aligned}
$$

Recalling that from (8) $\beta_{i}(x)=1$ only when $x \in S_{i}$, we can write (10) as

$$
\dot{x}=\sum_{i=1}^{r} \alpha_{i}(x)\left(A_{i}(p)-B_{i}(p) K_{i}\right) x .
$$

The state feedback fuzzy system (11) is recognized as an aggregation of $r$ local feedback uncertain systems described in a polytopic form.

\subsubsection{Guaranteed cost control design}

In this section, we propose a robust control design in terms of the optimal quadratic guaranteed cost problem as in Costa and Oliveira (2002). This approach is based on the local stability of each feedback fuzzy system of (11) in the subspace of the state space. In order to obtain a systematic control design, we formulate the problem in the context of the convex analysis using LMI's. 
Definition 1 The $i$ th feedback uncertain linear system of (11) is said to be asymptotically stable, if there exists a stabilizing control $u=-K_{i} x, i=1,2, \ldots, r$, such that an upper bound on the quadratic performance index

$$
C_{i}\left(x_{0}, u\right)=\int_{0}^{\infty}\left(x^{T} Q_{i} x+u^{T} R_{i} u\right) d t, x_{0} \in S_{i}^{\circ},
$$

along the system trajectory is minimized, with $Q_{i} \in \mathbb{R}^{n \times n}$, $R_{i} \in \mathbb{R}^{m \times m}, Q_{i}>0$, and $R_{i}>0$ weighting symmetric matrices which are chosen to yield the desired performance.

Definition 2 If there exist a stabilizing control law $u=$ $-K_{i} x, i=1,2, \ldots, r$ and a positive scalar $\hat{C}_{i}$, such that, $C_{i}\left(x_{0}, u\right) \leq \hat{C}_{i}$ along the system trajectory, then $\hat{C}_{i}$ is a guaranteed cost and $u$ is a guaranteed control law.

Proposition 1 Consider the $i$ th uncertain linear system of (4), control law $u=-K_{i} x, i=1,2, \ldots, r$ and cost performance (12). If there exist symmetric positive definite matrices $X_{i}$ and matrices $Y_{i}, i=1,2, \ldots, r$ of appropriate dimensions satisfying the LMI's

$$
\begin{array}{r}
Q_{i}>0, R_{i}>0, X_{i}>0, \\
{\left[\begin{array}{ll}
U_{i k} & Z_{i} \\
Z_{i}^{T} & -W
\end{array}\right]<0,} \\
\forall i=1,2, \ldots, r ; k=1,2, \ldots, v,
\end{array}
$$

where

$$
\begin{aligned}
& U_{i k}=X_{i} A_{i k}^{T}+A_{i k} X_{i}-Y_{i}^{T} B_{i k}^{T}-B_{i k} Y_{i}, \\
& Z_{i}=\left[\begin{array}{ll}
X_{i} Q_{i}^{1 / 2} & Y_{i}^{T} R_{i}^{1 / 2}
\end{array}\right], \\
& W=\operatorname{diag}\left\{\begin{array}{ll}
I_{n}, & I_{m}
\end{array}\right\}, \\
& X_{i}=P_{i}^{-1}, \\
& Y_{i}=K_{i} X_{i},
\end{aligned}
$$

then $u=-K_{i} x$, with $K_{i}=Y_{i} X_{i}^{-1}, i=1,2, \ldots, r$ is a guaranteed control law and the cost given by $\hat{C}_{i}=$ $x_{0}^{T} X_{i}^{-1} x_{0}$ is a guaranteed cost for the $i$ th feedback uncertain system of (11).

Proof: Consider a local quadratic Lyapunov function candidate as

$$
V_{i}(x)=x^{T} P_{i} x,
$$

which is a continuous-time function along the trajectory of (11) in the subspace $S_{i}^{\circ}$. Taking its derivatives, it results

$$
\begin{aligned}
\dot{V}_{i}(x)= & \dot{x}^{T} P_{i} x+x^{T} P_{i} \dot{x}, \\
= & x^{T}\left\{\alpha _ { i } ( x ) \left[\left(A_{i}(p)-B_{i}(p) K_{i}\right)^{T} P_{i}\right.\right. \\
& \left.\left.+P_{i}\left(A_{i}(p)-B_{i}(p) K_{i}\right)\right]\right\} x \\
= & x^{T}\left\{\sum _ { k = 1 } ^ { v } \alpha _ { i } ( x ) \eta _ { k } ( p ) \left[\left(A_{i k}-B_{i k} K_{i}\right)^{T} P_{i}\right.\right. \\
& \left.\left.+P_{i}\left(A_{i k}-B_{i k} K_{i}\right)\right]\right\} x .
\end{aligned}
$$

Now assume that there exist symmetric positive definite matrices $P_{i}=X_{i}^{-1}$ and matrices $K_{i}=Y_{i} X_{i}^{-1}$ satisfying LMI's in (13). Then, using the Schur complement (Boyd et al., 1994) after performing some algebraic manipulations, (13) can be reduced to

$$
\begin{array}{r}
\left(A_{i k}-B_{i k} K_{i}\right)^{T} P_{i}+P_{i}\left(A_{i k}-B_{i k} K_{i}\right) \\
+Q_{i}+K_{i}^{T} R_{i} K_{i}<0 \\
i=1,2, \ldots, r, k=1,2, \ldots, v .
\end{array}
$$

Using $\sum_{k=1}^{v} \eta_{k}(p)=1$, after some algebraic manipulations, (16) can be written as

$$
\begin{array}{r}
\sum_{k=1}^{v} \eta_{k}(p)\left[\left(A_{i k}-B_{i k} K_{i}\right)^{T} P_{i}+P_{i}\left(A_{i k}-B_{i k} K_{i}\right)\right] \\
+Q_{i}+K_{i}^{T} R_{i} K_{i}<0 \\
i=1,2, \ldots, r .
\end{array}
$$

Using (17) in (15), as $\alpha_{i}(x) \geq 0$, we have $\dot{V}_{i}(x)<0$, $\forall x \neq 0, x \in S_{i}^{\circ}$. Now, substituting (9) in (12) and using the fact that $\beta_{i}(x) \beta_{j}(x)=0, i \neq j, i, j=1,2, \ldots, r$ and $\beta_{i}(x) \beta_{i}(x)=1$, for $x \in S_{i}^{\circ}$ it results

$$
\begin{aligned}
C_{i}\left(x_{0}, u\right) & <\int_{0}^{\infty} x^{T}\left(Q_{i}+K_{i}^{T} R_{i} K_{i}\right) x d t \\
& <\int_{0}^{\infty} \dot{V}_{i}(x) d t \\
& =x_{0}^{T} P_{i} x_{0} .
\end{aligned}
$$

The results then follows by Definitions 1 and 2 .

The optimal quadratic guaranteed cost control problem involves the minimization of the cost bounds given by $\hat{C}_{i}=$ $x_{0}^{T} P_{i} x_{0}, i=1,2, \ldots, r$ which depends on the initial condition $x_{0} \in S_{i}^{\circ}$. To remove this dependence on $x_{0}$ one may assume it is a zero mean random variable satisfying $E\left[x_{0}^{T} x_{0}\right]=1$ and consider the minimization of $\operatorname{Tr}\left(P_{i}\right)$ as $E\left[C_{i}\right] \leq E\left[x_{0}^{T} x_{0}\right]=\operatorname{Tr}\left(P_{i}\right)$, with $E[\cdot]$ the expectancy operator and $\operatorname{Tr}(\cdot)$ is the trace (Jadbabaie et al., 1998b; Petersen and Macfarlane, 1994). Instead, we construct an optimization problem for the guaranteed cost control by minimizing an upper bound on the guaranteed cost $\hat{C}_{i}$.

Lemma 1 If $\hat{C}_{i}$ is a guaranteed cost for the ith state feedback uncertain linear system of (11) under performance in$\operatorname{dex}(12)$ then for $x_{0} \in S_{i}^{\circ}$

$$
\hat{C}_{B i}=\lambda_{\max }\left[X_{i}^{-1}\right]\left\|x_{0}\right\|^{2},
$$

$i=1,2, \ldots, r$, is a guaranteed cost for the ith state feedback uncertain linear system of (11) and an upper bound for (12), with $\lambda_{\max }[\cdot]$ the maximum eigenvalue and $\|\cdot\|$ the Euclidean vector norm. 
Proof: Using singular value decompositions the result follows straightforward.

Using both Proposition 1 and Lemma 1, we can construct the generalized eigenvalue problem (GEVP) (Boyd et al., 1994) for the guaranteed cost control design as

$$
\min _{X_{i}, Y_{i}} \gamma_{i} \text { subject to } I_{n} \leq \gamma_{i} X_{i} \text { and (13). }
$$

If (20) is feasible, we have $\gamma_{i}>\lambda_{\max }\left[X_{i}^{-1}\right]$ and $K_{i}=$ $Y_{i} X_{i}^{-1}, i=1,2, \ldots, r$.

\section{STABILITY ANALYSIS}

In Section 2 an approach to obtain the feedback gains and the quadratic Lyapunov functions associated is presented. Now, we establish a condition for the global stability of the feedback fuzzy system (11) by considering a class of piecewise quadratic Lyapunov functions and the fuzzy switching control proposed.

Theorem 2 The equilibrium $x=0$ of the globalfeedback TS fuzzy system with uncertainties (11) is asymptotically stable in the large if each uncertain linear system of (4) is locally stabilizable by the fuzzy switching controller (9) with $\beta_{i}(\cdot)$ as defined in (8) and $K_{i}$ resulting from (20), which is time continuous in the open subspace $S_{i}^{\circ}$.

Proof: Let

$$
V(x)=\sum_{i=1}^{r} \beta_{i}(x) V_{i}(x)
$$

be a piecewise quadratic Lyapunov function candidate with $V_{i}(x)$ as in (14) and $P_{i}=X_{i}^{-1}, X_{i}$ resulting from (20) for each subspace $S_{i}^{\circ}$ of the state space. In order to evaluate the derivative of (21) along the system trajectory, we replace $\dot{V}(\cdot)$ by the Dini derivative $D^{*} V(\cdot)$, where the superscript * in $D V(\cdot)$ represents any of the four Dini derivatives (Rouche et al., 1977). At any point where $\dot{V}(\cdot)$ exists, all four Dini derivatives have a common value equal to the derivative $\dot{V}(\cdot)$ at that point. See Apendix B for more details on the Dini derivatives. Let us consider the stability at the switching time. In the sequence, we use $x(t)$ to emphasize the analysis.

Suppose that for some particular time $t, x(t) \in S_{i}^{\circ}$ which yields $\beta_{i}(x(t))=1$ using (8). Also, suppose that the system equilibrium $x(t)=0$ does not exclusively belong to $S_{i}^{\circ}$, otherwise there might be no switching of controllers. After a period of time, a switching occurs, say at $t=t_{1}$, and $x(t)$ leaves the subspace $S_{i}^{\circ}$ and enters the $\ell$ th subspace $S_{\ell}^{\circ}$. We can thus write $\lim _{t \rightarrow t_{1}^{-}} \beta_{i}(x(t))=\beta_{i}\left(x\left(t_{1}\right)\right)=1$ and $\lim _{t \rightarrow t_{1}^{+}} \beta_{\ell}(x(t))=\beta_{\ell}\left(x\left(t_{1}\right)\right)=1$. The corresponding upper and lower Dini derivatives of (21) in the transition region $\partial S_{i \ell}$ are thus as

$$
\begin{gathered}
D^{+} V\left(x\left(t_{1}\right)\right)=D_{+} V\left(x\left(t_{1}\right)\right)=\underset{\substack{\epsilon \rightarrow 0^{+} \\
0<t-t_{1} \leq \epsilon}}{\sup } \\
\frac{1}{t-t_{1}} \sum_{\ell=1}^{r}\left[\beta_{\ell}(x(t)) V_{\ell}(x(t))-\beta_{\ell}\left(x\left(t_{1}\right)\right) V_{\ell}\left(x\left(t_{1}\right)\right)\right],
\end{gathered}
$$

$$
\begin{gathered}
D^{-} V\left(x\left(t_{1}\right)\right)=D_{-} V\left(x\left(t_{1}\right)\right)=\lim _{\substack{\epsilon \rightarrow 0^{-} \\
\epsilon \leq t-t_{1}<0}} \sup \\
\frac{1}{t-t_{1}} \sum_{i=1}^{r}\left[\beta_{i}(x(t)) V_{i}(x(t))-\beta_{i}\left(x\left(t_{1}\right)\right) V_{i}\left(x\left(t_{1}\right)\right)\right] .
\end{gathered}
$$

To have $V(\cdot)$ decreasing along the system trajectory any of the four Dini derivatives must be negative definite on the open subspace $S_{i}^{\circ}$ (see Corollary 7 in Appendix B). By the switching scheme (8), $D^{+} V(\cdot)$ in (22) is equal to $\dot{V}_{\ell}(\cdot)$ which is negative definite as $x \in S_{\ell}^{\circ}$. Then, as $\beta_{i}(\cdot)$ is a crisp function associated to each subspace $S_{i}^{\circ}, i=1,2, \ldots, r$, we can write

$$
\begin{aligned}
D^{+} V(x(t)) & =\sum_{i=1}^{r} \beta_{i}(x) \dot{V}_{i}(x) \\
& =\sum_{i=1}^{r} \beta_{i}(x)\left[\dot{x}^{T} P_{i} x+x^{T} P_{i} \dot{x}\right]
\end{aligned}
$$

Now, using (11) we can write (24) as

$$
\begin{array}{r}
D^{+} V(x(t))=\sum_{i=1}^{r} \beta_{i}(x) x^{T}\left\{\sum_{i=1}^{r} \sum_{k=1}^{v} \alpha_{i}(x) \eta_{k}(p)\right. \\
\left.\left[\left(A_{i k}-B_{i k} K_{i}\right)^{T} P_{i}+P_{i}\left(A_{i k}-B_{i k} K_{i}\right)\right]\right\} x,
\end{array}
$$

with $K_{i}=Y_{i} X_{i}^{-1}, X_{i}$ and $Y_{i}, i=1,2, \ldots, r$ resulting from (20). Using the proof of Proposition 1, for $\beta_{i}(\cdot)$ given by (8) we have $D^{+} V(x)<0, \forall x, x \neq 0$, which assures that system (11) is stable.

We proceed with the proof showing the stability in the large. Let us define

$$
-N_{i k}:=\left(A_{i k}-B_{i k} K_{i}\right)^{T} P_{i}+P_{i}\left(A_{i k}-B_{i k} K_{i}\right),
$$

where $N_{i k}=N_{i k}^{T}, N_{i k}>0$. For $x \in S_{i}^{\circ}, x \neq 0$, $i=1,2, \ldots, r$, we have $\dot{V}_{i}(x)<-\rho V_{i}(x)$ where $\rho$ is a positive number defined by $\rho:=\min _{i}\left\{\lambda_{\min }\left[N_{i k}\right] / \lambda_{\max }\left[P_{i}\right]\right\}$, $\lambda_{\min }[\cdot]$ and $\lambda_{\max }[\cdot]$ denote the minimum and maximum 
eigenvalues, respectively. By the well-known GronwallBellman lemma (Khalil, 1996) we can show that for $x_{0} \in S_{i}^{\circ}$, $V_{i}(x) \leq V_{i}\left(x_{0}\right) e^{-\rho t}$. Thus, from (24), it follows that

$$
\begin{aligned}
D^{+} V(x(t)) & =\sum_{i=1}^{r} \beta_{i}(x) \dot{V}_{i}(x) \\
& \leq-\rho \sum_{i=1}^{r} \beta_{i}(x) V_{i}(x) \\
& \leq-\rho \sum_{i=1}^{r} \beta_{i}\left(x_{0}\right) V_{i}\left(x_{0}\right) e^{-\rho t} \\
& =-\rho V\left(x_{0}\right) e^{-\rho t}
\end{aligned}
$$

as $\beta_{i}(\cdot)$ is a crisp function associated to each subspace $S_{i}^{\circ}$, $i=1,2, \ldots, r$, which completes the proof.

Remark 1 In order to compare the results given, we include in Appendix C, a fuzzy blending control approach which is also formulated as an optimal quadratic guaranteed cost control problem but adopting a common quadratic Lyapunov function (Arrifano and Oliveira, 2002).

Remark 2 To consider the stability of an uncertain nonlinear system for the case the origin $x=0$ is not the equilibrium, one should perform a change of coordinates to make it the equilibrium, before designing the fuzzy switching control (9) with $\beta_{i}(\cdot)$ as defined in (8). This change of coordinates is important because real systems, in general, have equilibrium different from the origin and the control design proposed considers asymptotic stability around the equilibrium $x=0$.

\section{SIMULATION RESULTS}

In this section, the usefulness of the switching fuzzy control is illustrated. We consider the stabilization of a magnetic suspension system and a mass-spring-damper system using the optimal quadratic guaranteed cost control. A feasible solution for the latter system can be obtained with the blending fuzzy control by means of a common Lyapunov function (see Appendix C) but no feasible solution is found for the former system.

Example 3 Nonlinear magnetic suspension system. We consider the same example as in Costa and Oliveira (1999), a nonlinear magnetic suspension system depicted by

$$
\begin{aligned}
& \dot{x}_{r 1}=x_{r 2} \\
& \dot{x}_{r 2}=g-\left(\frac{L_{b o}\left(x_{r 1}\right)}{2 a m}\right)\left(\frac{x_{r 3}}{1+\left(x_{r 1}\right) / a}\right)^{2} \\
& \dot{x}_{r 3}=-\left(\frac{R_{b}}{L_{b}}\right) x_{r 3}+\left(\frac{1}{L_{b}}\right) u_{r},
\end{aligned}
$$

Table 1: Magnetic suspension system parameters.

\begin{tabular}{cccccc}
\hline$g$ & $M$ & $R_{b}$ & $L_{b}$ & $L_{b 0}$ & $a$ \\
\hline 9.81 & $2.26 \times 10^{-2}$ & 19.91 & 470 & 0.0245 & $6.07 \times 10^{-3}$
\end{tabular}

where $x_{r 1}$ is the ball vertical position [m], $x_{r 2}$ is the ball vertical speed $[\mathrm{m} / \mathrm{s}], x_{r 3}$ is the coil current [A], $u_{r}$ is the coil applied voltage $[\mathrm{V}], g$ is the acceleration due to gravity $\left[\mathrm{m} / \mathrm{s}^{2}\right]$, $m$ is the ball mass $[\mathrm{Kg}], L_{b}$ is the coil inductance $[\mathrm{H}], R_{b}$ is the coil resistance [ $\Omega], a$ is a constant [m], and $L_{b 0}$ expresses the relationship between the inductance and the ball vertical position $[\mathrm{H}]$. Table 1 shows the numerical values of physical parameters. Note that the equilibrium of (28) $\left(x_{e}, u_{e}\right)$ is not the origin. As mentioned in Remark 2, it is necessary to perform a change of coordinates to bring the equilibrium of the system to the origin. For this purpose, we adopt $z=x_{r}-x_{e}$ and $v=u-u_{e}$, with $x_{e}=\left[\begin{array}{lll}0.010 & 0 & 0.8775\end{array}\right]$ and $u_{e}=17.4621$ the equilibrium of (28). Using these new coordinates, we may write (28) as

$$
\begin{aligned}
& \dot{z}_{1}=z_{2}+x_{e 2} \\
& \dot{z}_{2}=g-\left(\frac{L_{b 0}\left(z_{1}+x_{e 1}\right)}{2 a m}\right)\left(\frac{z_{3}+x_{e 3}}{1+\left(z_{1}+x_{e 1}\right) / a}\right)^{2} \\
& \dot{z}_{3}=-\left(\frac{R_{b}}{L_{b}}\right)\left(z_{3}+x_{e 3}\right)+\left(\frac{1}{L_{b}}\right)\left(v+u_{e}\right) .
\end{aligned}
$$

The uncertain linear systems are obtained using the linearization formula given in Appendix A considering $L_{b 0}$ as the uncertain parameter with deviations of about $\pm 80 \%$ from its nominal value. Adopting $r=2$ as the number of linearization points chosen and $\bar{x}_{(r=1)}=\left[\begin{array}{lll}0.005 & 0 & 0.6045\end{array}\right]$ and
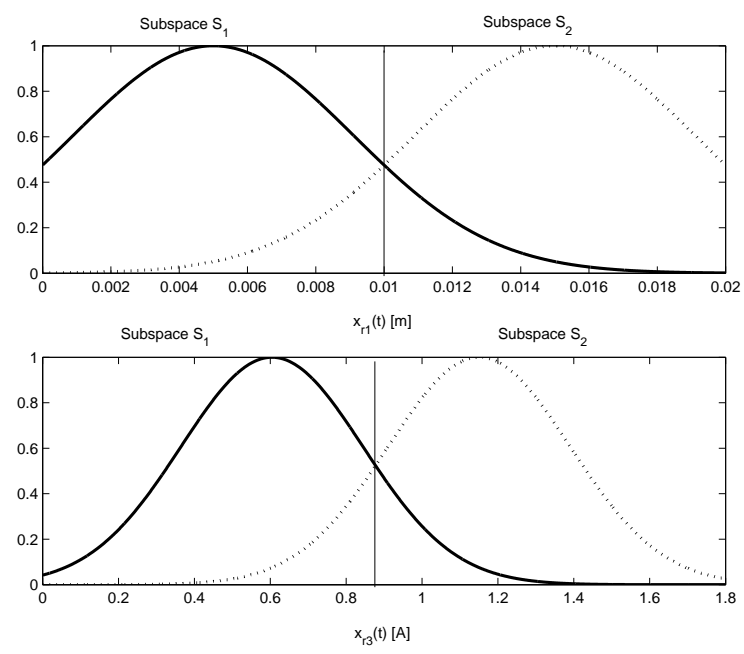

Figure 1: Membership functions adopted with “-” the $F_{j}^{1}\left(x_{j}\right)$ and “..." $F_{j}^{2}\left(x_{j}\right)$ 
$\bar{x}_{(r=2)}=\left[\begin{array}{lll}0.015 & 0 & 1.1505\end{array}\right]$ as the linearization points, we found the matrices representing the extreme linearized systems of each vertex system as

$$
\begin{gathered}
A_{11}=10^{3} \times\left[\begin{array}{rrr}
0 & 0.001 & 0 \\
1.2676 & 0 & -0.0334 \\
0 & 0 & -0.0423
\end{array}\right], B_{11}=\left[\begin{array}{r}
0 \\
0 \\
2.124
\end{array}\right], \\
A_{12}=10^{3} \times\left[\begin{array}{rrr}
0 & 0.001 & 0 \\
2.7677 & 0 & -0.0305 \\
0 & 0 & -0.0423
\end{array}\right] ; B_{12}=B_{11}, \\
A_{21}=10^{3} \times\left[\begin{array}{rrr}
6.9881 & 0 & -0.0583 \\
0 & 0 & -0.0423
\end{array}\right] ; B_{21}=B_{11}, \\
A_{22}=10^{3} \times\left[\begin{array}{rrr}
0 \\
0 & 0.001 & 0 \\
1.8819 & 0 & -0.0115 \\
0 & 0 & -0.0423
\end{array}\right] ; B_{22}=B_{11} .
\end{gathered}
$$

Figure 1 shows the membership functions adopted for $x_{r 1} \in$ $[0,0.020]$ and $x_{r 3} \in[0,1.5]$. Following, we present simulation results which are organized in two cases: (Case 1) $L_{b 0}\left(x_{r 1}\right)=L_{b 0}$ as in Costa and Oliveira (1999) and (Case 2) $L_{b 0}\left(x_{r 1}\right)=L_{b 0}\left(0.85+0.5 /\left(1+x_{r 1} / a\right)\right)$, with $L_{b 0}=0.0245 H$, the nominal value for $L_{b 0}(\cdot)$. The proposed approach is systematically accomplished by using the Matlab LMI solver as well as the ordinary differential equation (ODE) solver. We adopt the initial condition as $x_{0}=$ [0.005 00.6045$]$.

In Case 1 we adopt the weighting matrices

$$
Q_{i}=\left[\begin{array}{ccc}
10^{6} & 0 & 0 \\
0 & 1 & 0 \\
0 & 0 & 1
\end{array}\right] \text { and } R_{i}=0.05, \text { for } i=1,2
$$

as in Costa and Oliveira (1999). In Case 2 we adopt the weighting matrices

$$
\begin{gathered}
Q_{1}=\left[\begin{array}{ccc}
10^{5} & 0 & 0 \\
0 & 1 & 0 \\
0 & 0 & 1
\end{array}\right] ; Q_{2}=\left[\begin{array}{ccc}
5 \times 10^{4} & 0 & 0 \\
0 & 1 & 0 \\
0 & 0 & 1
\end{array}\right] \\
R_{1}=0.001 \text { and } R_{2}=0.05 .
\end{gathered}
$$

The numerical results are summarized in Table 2 and 3 for Cases 1 and 2, respectively. Figures 2 and 3 show the responses of system (28) for Case 1 and Figures 4 and 5 present the responses for Case 2. Using the fuzzy switching control approach, the switching in the control law can be flattered by adjusting the width and the type of the membership functions adopted as well as the matrices $Q_{i}$ and $R_{i}, i=1,2, \ldots, r$. Therefore, the proposed solution can yield smother solutions than the one given in Costa and Oliveira (1999) using attraction domains for the switching control scheme. Other characteristic of this approach is that the switching is related to the smaller value of the associated Lyapunov function when the
Table 2: (Case 1) Numerical results of the control design.

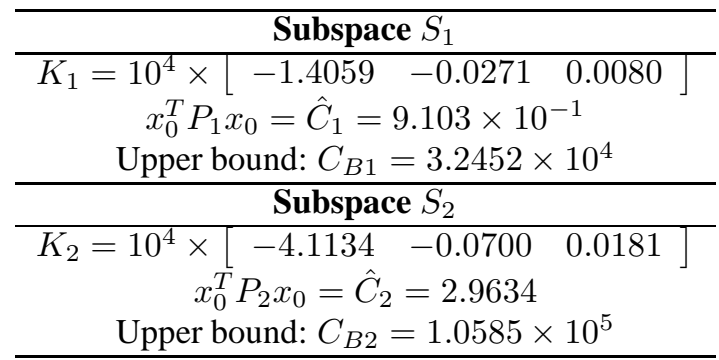

Table 3: (Case 2) Numerical results of the control design.

\begin{tabular}{c}
\hline Subspace $S_{1}$ \\
\hline$K_{1}=10^{4} \times[-2.1864-0.03880 .0100]$ \\
$x_{0}^{T} P_{1} x_{0}=\hat{C}_{1}=7.15 \times 10^{-2}$ \\
Upper bound: $C_{B 1}=1.7002 \times 10^{3}$ \\
\hline Subspace $S_{2}$ \\
\hline$K_{2}=10^{4} \times[-3.5301-0.06080 .0167]$ \\
$x_{0}^{T} P_{2} x_{0}=\hat{C}_{2}=1.5781$ \\
Upper bound: $C_{B 2}=7.5344 \times 10^{3}$ \\
\hline
\end{tabular}

state is on the boundary of the defined subspaces, but the system solution always returns to the subspace that better represents the dynamics of the nonlinear system. After the state vector enters the subspace where the equilibrium point $x_{e}$ is, the switching occurs if the resulting Lyapunov functions have not reached the origin or if the system are subjected to perturbations.

Example 4 Nonlinear mass-spring-damper system. We consider now the same example as in Tanaka et al. (1996), a nonlinear mass-spring-damper system with an uncertain parameter, which is described as

$$
M \ddot{y}+g(y, \dot{y})+f(y)=\phi(\dot{y}) u,
$$

where $M$ is the mass $[\mathrm{Kg}], u$ is the force [N], $y$ is the vertical position [m], $\dot{y}$ is the speed [m/s], $g(y, \dot{y})=c_{1} y+c_{2} \dot{y}$, $f(y)=c$, and $\phi(\dot{y})=1+c_{5} \dot{y}^{3}$ are the nonlinear or uncertain terms with respect to the spring, the damper and the input system, respectively. The control purpose is to achieve the equilibrium $(x, u)=(0,0)$ with the minimization of an upper bound on the guaranteed cost. Considering the parameters $M=1, c \in\left[c_{3}, c_{4}\right], c_{1}=0, c_{2}=1, c_{3}=0.5$, $c_{4}=1.81$ and $c_{5}=0.13$, and defining $x:=\left[\begin{array}{ll}y & \dot{y}\end{array}\right]^{T}$, we can write (30) in the state space representation

$$
\left[\begin{array}{l}
\dot{x}_{1} \\
\dot{x}_{2}
\end{array}\right]=\left[\begin{array}{rr}
-1 & -c \\
1 & 0
\end{array}\right]\left[\begin{array}{l}
x_{1} \\
x_{2}
\end{array}\right]+\left[\begin{array}{c}
1+0.13 x_{1}^{3} \\
0
\end{array}\right] u \text {. }
$$



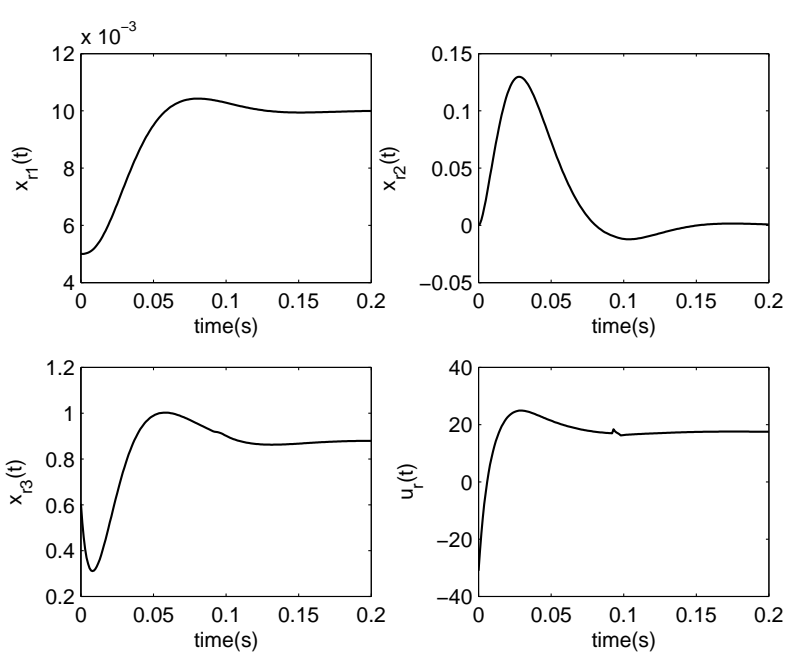

Figure 2: (Case 1) Magnetic suspension system state $x_{r}$ and control $u$.

As system (31) presents one uncertain parameter, we have two vertexes in the polytopic description. We adopt $r=2$ and again we obtain the uncertain linear systems using the linearization formula for the following linearization points $\bar{x}$ : $\bar{x}_{(r=1)}=\left[\begin{array}{ll}1.9740 & 0\end{array}\right]^{T}$ and $\bar{x}_{(r=2)}=\left[\begin{array}{ll}-1.9740 & 0\end{array}\right]^{T}$, which gives

$$
\begin{aligned}
& A_{11}=\left[\begin{array}{rr}
-1 & -0.5 \\
1 & 0
\end{array}\right], B_{11}=\left[\begin{array}{c}
1.4387 \\
0
\end{array}\right], \\
& A_{12}=\left[\begin{array}{rr}
-1 & -1.81 \\
1 & 0
\end{array}\right], B_{12}=B_{11}, \\
& A_{21}=A_{11}, B_{21}=\left[\begin{array}{c}
0.5613 \\
0
\end{array}\right], \\
& A_{22}=A_{12}, \quad B_{22}=B_{21} .
\end{aligned}
$$

The performance of the proposed approach can be verified adopting $\alpha_{1}(x)=0.5+x_{1}^{3} / 6.75, \alpha_{2}(x)=0.5-x_{1}^{3} / 6.75$ and $c=1.155+0.655 \cos \left(3 x_{2}^{10 \sin \left(x_{1}\right)}\right)$ for $x_{1} \in[-1.5,1.5]$ and $x_{2} \in[-1.5,1.5]$. The control design is systematically developed by solving the optimization problem (50). We choose $Q=I_{2}$ and $R=0.07$ for both rules and adopt initial condition $x_{0}=\left[\begin{array}{ll}-0.5 & -1.0\end{array}\right]^{T}$. Using the Matlab LMI solver, we obtain the main results summarized in Table 4. Figure 6 shows the feedback uncertain nonlinear system responses. The proposed approach is comparable to the one given in Tanaka et al. (1996). Its advantage is that it follows a systematic procedure and minimizes an upper bound on the quadratic performance cost.

\section{CONCLUSION}

In this paper we propose a fuzzy switching control design to stabilize a class of uncertain nonlinear systems represented
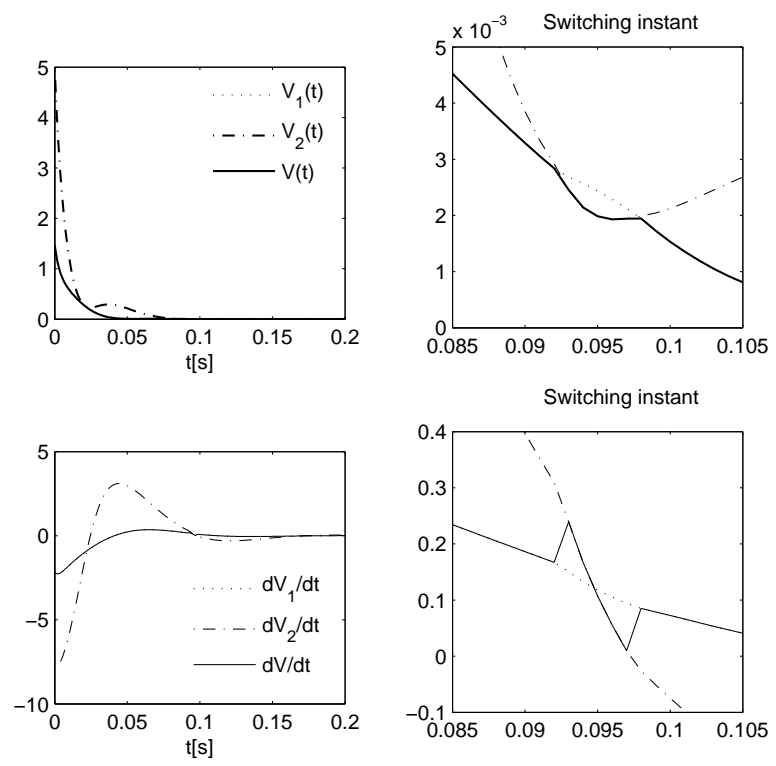

Figure 3: (Case 1) Lyapunov functions and their derivatives along the system trajectory.

Table 4: Numerical results of the control design.

\begin{tabular}{c}
\hline$K_{1}=\left[\begin{array}{ll}1.60 & 1.08\end{array}\right]$ \\
$K_{2}=\left[\begin{array}{ll}4.10 & 2.77\end{array}\right]$ \\
$x_{0}^{T} P x_{0}=\hat{C}=1.7885$ \\
Upper bound: $C_{B}=1.7947$ \\
\hline
\end{tabular}

by uncertain TS fuzzy systems. A sufficient condition for the stability of the state feedback fuzzy system is given in terms of a piecewise quadratic Lyapunov function. The control design is formulated in the context of the guaranteed cost control problem with the minimization of an upper bound on the guaranteed cost. The fuzzy switching control produces less conservative results than the fuzzy blending control approach which uses a common quadratic Lyapunov function. In addition, the approach given may be applied to highly nonlinear systems, where available robust control techniques are not successful. The proposed approach yields a computationally tractable solution to the control design in the context of LMI's. Further, different control techniques to design the local controllers can also be explored using the framework presented. 

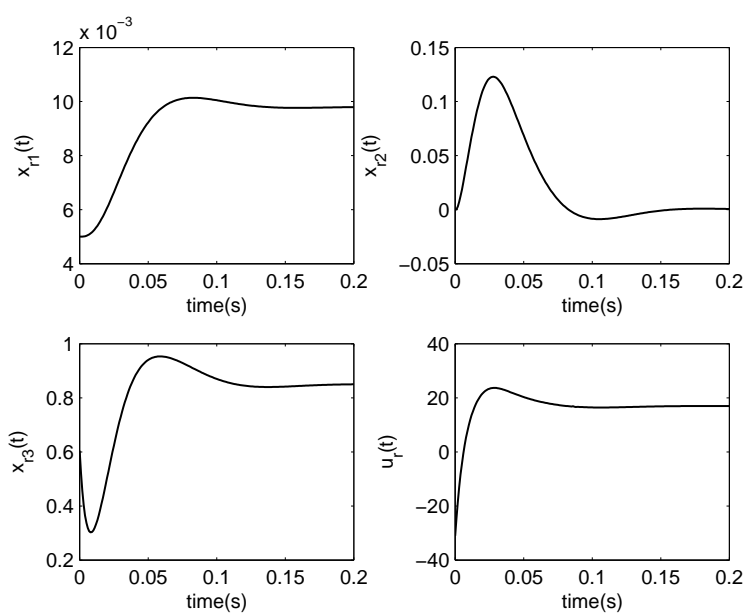

Figure 4: (Case 2) Magnetic suspension system state $x_{r}$ and control $u_{r}$.

\section{ACKNOWLEDGEMENTS}

The authors thank the anonymous referees by the useful comments and suggestions. This work was supported by the Fundação do Amparo à Pesquisa do Estado de São Paulo (FAPESP) under grant 00/05060-1 and by the Conselho de Desenvolvimento Científico e Tecnológico (CNPq) under grant 301982/03-1.

\section{A LOCAL LINEAR APPROXIMATIONS OF THE UNCERTAIN NONLINEAR SYS- TEMS}

Consider the uncertain nonlinear systems in its polytopic description as defined in (2). Following, we present the linearization formula used to obtain the uncertain linear approximations of the nonlinear functions which are the vertexes of the polytope. For this purpose, let $\bar{x}$ denote a linearization point, which is not necessarily an equilibrium point. The objective is to obtain matrices $A_{k}$ and $B_{k}$ such that in the vicinity of $\bar{x}$ we have

$$
f_{k}(x)+g_{k}(x) u \approx A_{k} x+B_{k} u
$$

and

$$
f_{k}(\bar{x})+g_{k}(\bar{x}) u \approx A_{k} \bar{x}+B_{k} u,
$$

with $f_{k}(\cdot), g_{k}(\cdot), x$ and $u$ as defined before. Since $u$ is arbitrary, we have $g_{k}(\bar{x})=B_{k}$. Thus, the procedure reduces to finding matrices $A_{k}$ such that, in the vicinity of $\bar{x}$, we have

$$
f_{k}(x) \approx A_{k} x
$$

and

$$
f_{k}(\bar{x}) \approx A_{k} \bar{x}
$$
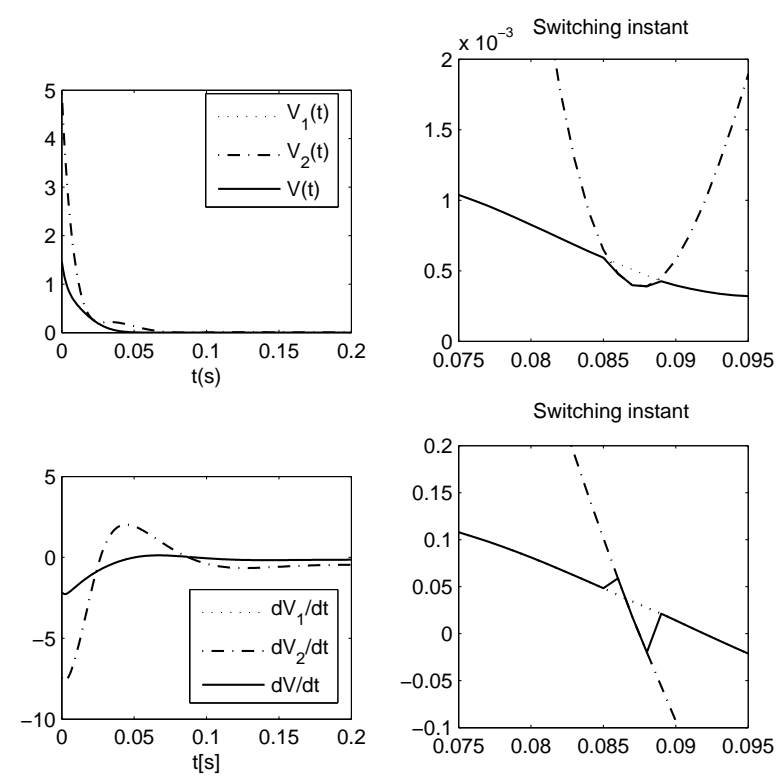

Figure 5: (Case 2) Lyapunov function and their derivatives along the system trajectory.

Following (Teixeira and Żak, 1999), let $a_{j k}^{T}$ denote the $j$ th row of matrix $A_{k}$. Then, conditions (32) and (33) can be written as

$$
f_{j k}(x) \approx a_{j k}^{T} x
$$

and

$$
f_{j k}(\bar{x}) \approx a_{j k}^{T} \bar{x}
$$

respectively, where $f_{j k}(\cdot): \mathbb{R}^{n} \rightarrow \mathbb{R}$ is the $j$ th component of $f_{k}(\cdot)$ for $j=1,2, \ldots, n$. Expanding the left hand side of (34) over $\bar{x}$ and neglecting the second and higher order terms we obtain

$$
f_{j k}(\bar{x})+\nabla^{T} f_{j k}(\bar{x})(x-\bar{x}) \approx a_{j k}^{T} x,
$$

where $\nabla f_{j k}(\cdot): \mathbb{R}^{n} \rightarrow \mathbb{R}^{n}$ is the gradient, a column vector of $f_{j k}(\cdot)$ computed with respect to $x$. Now, using (35) and (36), we have

$$
\nabla^{T} f_{j k}(\bar{x})(x-\bar{x}) \approx a_{j k}^{T}(x-\bar{x}),
$$

where $x$ is arbitrary but "close" to $\bar{x}$. Finally, we obtain a constant vector $a_{j k}$ as close as possible to $\nabla f_{j k}(\bar{x})$ satisfying $a_{j k}^{T} \bar{x}=f_{j k}(\bar{x})$ solving the constrained optimization problem $\min _{a_{j k}} E=\frac{1}{2}\left\|\nabla f_{j k}(\bar{x})-a_{j k}\right\|_{2}^{2}$ subjecto to $a_{i k}^{T} \bar{x}=f_{i k}(\bar{x})$.

According to Teixeira and Żak (1999), the first order conditions to solve this optimization problem are

$$
\nabla_{a_{j k}} E+\lambda \nabla_{a_{j k}}\left[a_{j k}^{T} \bar{x}-f_{j k}(\bar{x})\right]=0
$$



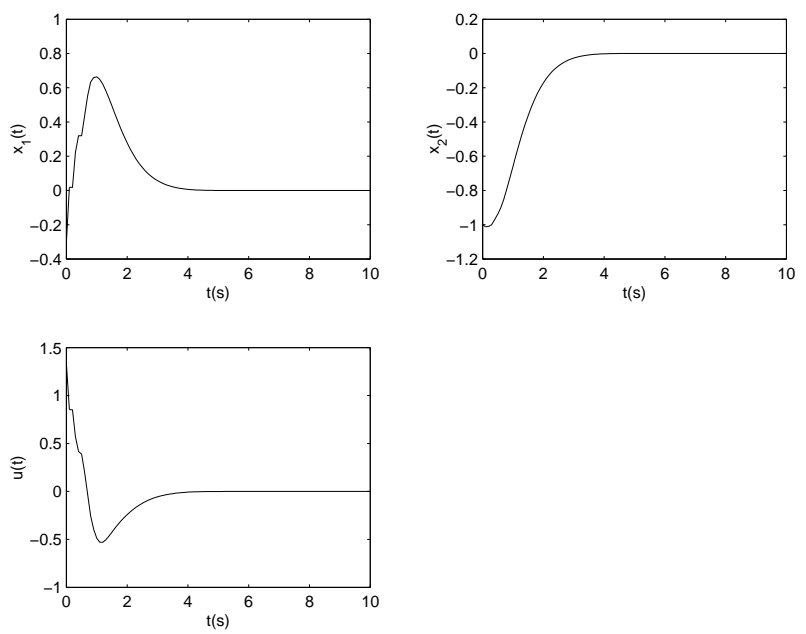

Figure 6: Mass-spring-damper system state $x$ and control $u$.

and

$$
a_{j k}^{T} \bar{x}=f_{j k}(\bar{x}),
$$

where $\lambda$ in (38) is the Lagrange multiplier and the subscript $a_{j k}$ in $\nabla_{a_{j k}}$ indicates that the gradient $\nabla$ is computed with respect to $a_{j k}$. Performing the required differentiation in (38), it yields

$$
a_{j k}-\nabla f_{j k}(\bar{x})+\lambda \bar{x}=0
$$

Pre-multiplying (40) by $\bar{x}^{T}$ and using (39), we obtain

$$
\lambda=\frac{\bar{x}^{T} \nabla f_{j k}(\bar{x})-f_{j k}(\bar{x})}{\|\bar{x}\|^{2}} .
$$

Now, substituting (41) in (40), we obtain

$$
a_{j k}=\nabla f_{j k}(\bar{x})+\frac{f_{j k}(\bar{x})-\bar{x}^{T} \nabla f_{j k}(\bar{x})}{\|\bar{x}\|^{2}} \bar{x}, \bar{x} \neq 0,
$$

which are the columns of the vertex matrix $A_{k}$. This formula produces linear approximations instead of affine approximations, which are in general obtained by the Taylor linearization formula given by

$$
A_{k}=\nabla f_{k}(\bar{x}):=\left.\frac{\partial f_{k}(x)}{\partial x}\right|_{x=\bar{x}},
$$

and the approximation of $f_{k}(x)$ around $\bar{x}$ is

$$
f_{k}(x) \approx f_{k}(\bar{x})+\nabla f_{k}(\bar{x})(x-\bar{x}) .
$$

Note that for $f_{k}(\bar{x}) \neq 0$, this approach produces affine systems instead of linear ones, as mentioned before. Using (42), several linear approximations of the uncertain linear system (2) can be obtained for different linearization points, even if these points are not equilibrium points.

\section{B THE DINI DERIVATIVES}

The Dini derivatives are a generalization of the classical derivative and inherit some important properties from it. Because the Dini derivatives are point-wise defined, they are more suited than some more modern approaches to generalize the concept of a derivative like Sobolev Space or Distributions. The Dini derivatives are defined as follows (Rouche et al., 1977).

Definition 3 Let $] a, b[\subset \mathbb{R}$ and consider a function $f:] a, b\left[\rightarrow \mathbb{R}\right.$ and a point $\left.t_{0} \in\right] a, b[$.

(i) Let $t_{0}$ be a limit point of $] a, b[\cap] t_{0},+\infty[$. Then the right-hand upper Dini derivate $D^{+}$of $f$ at $t_{0}$ is given by

$$
\begin{aligned}
& D^{+} f\left(t_{0}\right):=\limsup _{t \rightarrow t_{0}^{+}} \frac{f(t)-f\left(t_{0}\right)}{t-t_{0}}= \\
& \left.\lim _{\epsilon \rightarrow 0^{+}} \sup _{\substack{t \in] a, b[\cap] t_{0},+\infty\left[ \\
0<t-t_{0} \leq \epsilon\right.}} \frac{f(t)-f\left(t_{0}\right)}{t-t_{0}}\right),
\end{aligned}
$$

and the right-hand lower Dini derivate $D_{+}$of $f$ at $t_{0}$ is given by

$$
\begin{aligned}
& D_{+} f\left(t_{0}\right):=\liminf _{t \rightarrow t_{0}^{+}} \frac{f(t)-f\left(t_{0}\right)}{t-t_{0}}= \\
& \lim _{\epsilon \rightarrow 0^{+}}\left(\begin{array}{l}
\inf _{\substack{t \in] a, b[\cap] t_{0},+\infty\left[ \\
0<t-t_{0} \leq \epsilon\right.}} \frac{f(t)-f\left(t_{0}\right)}{t-t_{0}}
\end{array}\right)
\end{aligned}
$$

where $t \rightarrow t_{1}^{+}$means simply that one considers, in the limiting processes, only the values of $t>t_{1}$. A similar meaning is attached to $t \rightarrow t_{1}^{-}$.

(ii) Let $t_{0}$ be a limit point of $] a, b[\cap]-\infty, t_{0}[$. Then the left-hand upper Dini derivate $D^{-}$of $f$ at $t_{0}$ is given by

$$
\begin{aligned}
& D^{-} f\left(t_{0}\right):=\limsup _{t \rightarrow t_{0}^{-}} \frac{f(t)-f\left(t_{0}\right)}{t-t_{0}}= \\
& \lim _{\epsilon \rightarrow 0^{-}}\left(\begin{array}{l}
\sup _{\substack{t \\
t \in] a, b[\cap]-\infty, t_{0}\left[ \\
\epsilon \leq t-t_{0}<0\right.}} \frac{f(t)-f\left(t_{0}\right)}{t-t_{0}}
\end{array}\right),
\end{aligned}
$$

and the left-hand lower Dini derivate $D_{-}$of $f$ at $t_{0}$ is 
given by

$$
\begin{aligned}
& D_{-} f\left(t_{0}\right):=\liminf _{t \rightarrow t_{0}^{-}} \frac{f(t)-f\left(t_{0}\right)}{t-t_{0}}= \\
& \lim _{\epsilon \rightarrow 0^{-}}\left(\begin{array}{l}
\inf _{\substack{t \in] a, b[\cap] t_{0},+\infty\left[ \\
\epsilon \leq t-t_{0}<0\right.}} \frac{f(t)-f\left(t_{0}\right)}{t-t_{0}}
\end{array}\right)
\end{aligned}
$$

In the framework of the elementary calculus, if $f:] a, b[\rightarrow \mathbb{R}$ is a function from a non-empty open subset $] a, b[\subset \mathbb{R}$ into $\mathbb{R}$ and $\left.t_{0} \in\right] a, b\left[\right.$, then all four Dini derivatives $D^{+} f\left(t_{0}\right)$, $D_{+} f\left(t_{0}\right), D^{-} f\left(t_{0}\right)$, and $D_{-} f\left(t_{0}\right)$ of $f$ at the point $t_{0}$ exist. This means that if $] a, b[$ is a non-empty open interval, then the functions $D^{+} f, D_{+} f, D^{-} f$ and $\left.D_{-} f:\right] a, b[\rightarrow \overline{\mathbb{R}}$, where $\overline{\mathbb{R}}:=\mathbb{R} \cup\{-\infty\} \cup\{+\infty\}$, are all defined in the canonical form. In this case, the classical derivative $d f / d t:] a, b[\rightarrow \mathbb{R}$ exists, if and only if the Dini derivatives are all real valued and $D^{+} f=D_{+} f=D^{-} f=D_{-} f$.

Remark 3 We have the inequality for lim sup

$$
\limsup _{t \rightarrow t_{0}^{+}}[f(t)+g(t)] \leq \limsup _{t \rightarrow t_{0}^{+}} f(t)+\limsup _{t \rightarrow t_{0}^{+}} g(t)
$$

in which a derivative defined in this form is not a linear operation at all; notwithstanding, if the right-hand limit of the function $g$ exists, then

$$
\limsup _{t \rightarrow t_{0}^{+}}[f(t)+g(t)]=\limsup _{t \rightarrow t_{0}^{+}} f(t)+\lim _{t \rightarrow t_{0}^{+}} g(t) .
$$

These results also hold for lim inf.

The latter equality leads to the following lemma.

Lemma 5 Let $f$ and $g$ be real valued functions, the domains of which are subsets of $\mathbb{R}$ and let $D^{*} \in$ $\left\{D^{+} f, D_{+} f, D^{-} f, D_{-} f\right\}$ be a Dini derivative. Let $t_{0} \in \mathbb{R}$ be such that the Dini derivative $D^{*} f\left(t_{0}\right)$ is properly defined; that is $D^{*} f\left(t_{0}\right) \in \mathbb{R}$ and $g$ is differentiable at $t_{0}$ in the classical sense. Then

$$
D^{*}\left[f\left(t_{0}\right)+g\left(t_{0}\right)\right]=D^{*} f\left(t_{0}\right)+\frac{d g\left(t_{0}\right)}{d t} .
$$

Theorem 6 Let $I$ be a non-empty interval in $\mathbb{R}, C$ be a countable subset of $I$ and $f: I \rightarrow \mathbb{R}$ be a continuous function. Let $D^{*} \in\left\{D^{+} f, D_{+} f, D^{-} f, D_{-} f\right\}$ be a Dini derivative and let $J$ be an interval such that $D^{*} f(t) \in J$ for all $t \in I / C$. Then

$$
\frac{f\left(t_{1}\right)-f\left(t_{2}\right)}{t_{1}-t_{2}} \in J,
$$

for all $t_{1}, t_{2} \in I, t_{1} \neq t_{2}$.
Corollary 7 Let $I$ be a non-empty interval in $\mathbb{R}, C$ be a countable subset of $I, f: I \rightarrow \mathbb{R}$ be a continuous function, and $D^{*} \in\left\{D^{+} f, D_{+} f, D^{-} f, D_{-} f\right\}$ be a Dini derivative. Then

$D^{*} f(t) \geq 0$ for all $t \in I / C$ implies that $f$ is increasing on $I$,

$D^{*} f(t)>0$ for all $t \in I / C$ implies that $f$ is strictly increasing on $I$,

$D^{*} f(t) \leq 0$ for all $t \in I / C$ implies that $f$ is decreasing on $I$,

$D^{*} f(t)<0$ for all $t \in I / C$ implies that $f$ is strictly decreasing on $I$.

\section{FUZZY BLENDING CONTROL}

For the purpose of comparison, we present a fuzzy blending control which is also used to stabilize (4). This stabilizing control approach is given in terms of the PDC scheme and a common quadratic Lyapunov function using the guaranteed cost control optimization problem in the context of the convex analysis using LMI's (Arrifano and Oliveira, 2002).

According to the PDC scheme, a fuzzy blending control shares the same structure of (3) in its premise part. As in (4), this fuzzy control is also inferred as a weighted average of all feedback gains $K_{i}, i=1,2, \ldots, r$ which is given by

$$
u=-\sum_{i=1}^{r} \alpha_{i}(x) K_{i} x
$$

with $\alpha_{i}(\cdot)$ as in (5). In order to obtain the state feedback fuzzy system, we substitute (44) in (4), which gives

$$
\dot{x}=\sum_{i=1}^{r} \sum_{j=1}^{r} \alpha_{i}(x) \alpha_{j}(x)\left(A_{i}(p)+B_{i}(p) K_{j}\right) x .
$$

Defining $G_{i}(p):=A_{i}(p)-B_{i}(p) K_{i}$ and $H_{i j}(p):=A_{i}(p)-$ $B_{i}(p) K_{j}+A_{j}(p)-B_{j}(p) K_{i}, i, j=1,2, \ldots, r$, after some algebraic manipulations using $\sum_{i=1}^{r} \alpha_{i}(x)=1$, we can write (45) as

$$
\dot{x}=\sum_{i=1}^{r} \alpha_{i}^{2}(x) G_{i}(p) x+\sum_{i<j}^{r} \alpha_{i}(x) \alpha_{j}(x) H_{i j}(p) x .
$$

In (46), $\sum_{i<j}^{r}$ means, for instance for $r=3, \sum_{i<j}^{3} a_{i j} \Leftrightarrow$ $a_{12}+a_{13}+a_{23}$. 


\section{C.1 Guaranteed cost control design via LMI's}

In this section we summarize the optimal quadratic guaranteed cost control problem for the fuzzy blending control design.

Definition 4 The fuzzy system (4) is said to be stable if there exists a stabilizing control law as in (44) such that an upper bound on the quadratic performance index

$$
C\left(x_{0}, u\right)=\int_{0}^{\infty}\left(x^{T} Q x+u^{T} R u\right) d t,
$$

along the feedback fuzzy system trajectory is minimized with $Q \in \mathbb{R}^{n \times n}, R \in \mathbb{R}^{m \times m}, Q>0$, and $R>0$ weighting symmetric matrices which are chosen to yield the desired performance.

Definition 5 If there exist a stabilizing control law as in (44) and a positive scalar $\hat{C}$ such that $C\left(x_{0}, u\right) \leq \hat{C}$ along the feedback fuzzy system trajectory then $\hat{C}$ is a guaranteed cost and (44) is a guaranteed control law.

Proposition 2 Consider the fuzzy system (4), the fuzzy blending control (44) and the performance index (47). If there exist a common symmetric positive definite matrix $X$ and matrices $Y_{i}, i=1,2, \ldots, r$ of appropriate dimensions satisfying the LMI's

$$
\begin{aligned}
& Q>0, R>0, X>0, \\
& {\left[\begin{array}{ll}
U_{i k} & Z \\
Z^{T} & -W
\end{array}\right]<0,} \\
& \forall i=1,2, \ldots, r, k=1,2, \ldots, v \\
& {\left[\begin{array}{ll}
V_{i j k} & Z \\
Z^{T} & -W
\end{array}\right]<0,} \\
& \forall i<j, i, j=1,2, \ldots, r, k=1,2, \ldots, v,
\end{aligned}
$$

where

$$
\begin{aligned}
& U_{i k}=X A_{i k}^{T}+A_{i k} X-Y_{i}^{T} B_{i k}^{T}-B_{i k} Y_{i}, \\
& V_{i j k}=X A_{i k}^{T}+A_{i k} X-Y_{j}^{T} B_{i k}^{T}-B_{i k} Y_{j} \\
& +X A_{j k}^{T}+A_{j k} X-Y_{i}^{T} B_{j k}^{T}-B_{j k} Y_{i}, \\
& Z=\quad\left[\begin{array}{lllll}
X Q^{1 / 2} & Y_{1}^{T} R^{1 / 2} & Y_{2}^{T} R^{1 / 2} & \ldots & Y_{r}^{T} R^{1 / 2}
\end{array}\right], \\
& W=\operatorname{diag}\left\{I_{n}, \quad I_{m}, I_{m}, \ldots, I_{m}\right\} \\
& Y_{i}=K_{i} X \text {, } \\
& X=P^{-1} \text {. }
\end{aligned}
$$

then (44) with $K_{i}=Y_{i} X^{-1}, i=1,2, \ldots, r$ is a guaranteed control law and the cost given by $\hat{C}=x_{0}^{T} X^{-1} x_{0}$ is a guaranteed cost.

Proof: The proof can be obtained following the proof of Proposition 1, considering a common quadratic Lyapunov function candidate as

$$
V(x)=x^{T} P x,
$$

along the feedback fuzzy system trajectory.

As in Section 2.2.1, using both Proposition 2 and Lemma 1, we can construct the following GEVP for the guaranteed cost control design to the feedback fuzzy system (46):

$$
\min _{X, Y_{i}} \gamma \text { subject to } I_{n} \leq \gamma X \text { and (48). }
$$

If (50) is feasible, we have $\gamma>\lambda_{\max }\left[X^{-1}\right]$ and $K_{i}=$ $Y_{i} X^{-1}$ for $i=1,2, \ldots, r$.

\section{REFERENCES}

Arrifano, N. S. D. and Oliveira, V. A. (2002). Guaranteed cost fuzzy controllers for a class of uncertain nonlinear dynamic systems, XIV Congresso Brasileiro de Automática, pp. 1873-1877.

Boyd, S., Ghaoui, L. E., Feron, E. and Balakrishnan, V. (1994). Linear Matrix Inequalities in System and Control Theory, SIAM, Philadelphia, PA.

Cao, S. G., Rees, N. W. and Feng, G. (1997). Further results about quadratic stability of continuous-time fuzzy control systems, International Journal of Systems Science 4(28): 397-404.

Cao, S. G., Rees, N. W. and Feng, G. (2001). H $\infty$ control of uncertain fuzzy continuous-time systems, Fuzzy Sets and Systems 115(2): 171-190.

Costa, E. F. and Oliveira, V. A. (1999). Gain scheduled controllers for dynamic systems with sector nonlinearities, 14th IFAC World Congress, Vol. E, Beiging, China, pp. 357-362.

Costa, E. F. and Oliveira, V. A. (2002). On the design of guaranteed cost controllers for a class of uncertain linear systems, Systems \& Control Letters 46(1): 17-29.

Feng, G., Cao, S. G., Rees, N. W. and Chack, C. K. (1997). Design of fuzzy control systems with guaranteed stability, Fuzzy Sets and Systems 85(1): 1-10.

Jadbabaie, A., Abdallah, C. T., Farmularo, D. and Dorato, P. (1998b). Robust, non-fragile and optimal controller design via linear matrix inequalities, American Control Conference, Philadelphia, PA, pp. 2842-2846.

Jadbabaie, A., Jamshidi, M. and Titli, A. (1998a). Guaranteed cost design of continuous-time takagi-sugeno fuzzy controllers via linear matrix inequalities, IEEE International Conference on Fuzzy Systems, Vol. 1, Anchorage, AK, pp. 268-273. 
Johansson, M., Rantzer, A. and Arzén, K. (1998). Piecewise quadratic stability of fuzzy systems, IEEE Transactions on Fuzzy Systems 7(6): 713-722.

Khalil, H. (1996). Nonlinear Systems, Prentice-Hall, Upper Saddle River, NJ.

Lee, K. R., Jeung, E. T. and Park, H. B. (2001). Robust fuzzy $\mathbf{H} \infty$ control for uncertain nonlinear systems via state feedback: an LMI approach, Fuzzy Sets and Systems 120(1): 123-134.

Petersen, I. R. and Macfarlane, D. C. (1994). Optimal guaranteed cost control and filtering for uncertain linear systems, IEEE Transactions on Automatic Control 39(9): 1971-1977.

Rouche, N., habets, P. and Laloy, M. (1977). Stability Theory by Lyapunov's Direct Method, Springer-Verlag, New York, NY.

Takagi, T. and Sugeno, M. (1985). Fuzzy identification of systems and its application to modeling and control, IEEE Transactions on Systems, Man and Cybernetic 15(1): 116-132.

Tanaka, K., Hori, T., Yamafugi, K. and Wang, O. H. (1999). An integrated fuzzy control system design for nonlinear systems, 38th IEEE Conference on Decision and Control, Vol. 5, Phoenix, Arizona, pp. 4349-4354.

Tanaka, K., Ikeda, T., and Wang, H. O. (1997). Fuzzy control system design via LMI, American Control Conference, Vol. 5, Albuquerque, NM, pp. 2873-2877.

Tanaka, K., Ikeda, T. and Wang, H. O. (1996). Robust stabilization of a class of uncertain nonlinear systems via fuzzy control: quadratic stabilizability, $\mathbf{H}_{\infty}$ control theory and linear matrix inequalities, IEEE Transactions on Fuzzy Systems 4(1): 1-13.

Tanaka, K., Ikeda, T. and Wang, H. O. (1998). Fuzzy regulators and fuzzy observers: relaxed stability conditions and LMI-based designs, IEEE Transactions on Fuzzy Systems 8(2): 250-265.

Tanaka, K. and Wang, H. O. (2001). Fuzzy Control Systems Design and Analysis: a Linear Matrix Inequality Approach, John Wiley and Sons, New York, NY.

Teixeira, M. C. M., Pietrobom, H. C. and Assunção, E. (2000). Novos resultados sobre a estabilidade e controle de sistemas não-lineares utilzando modelos fuzzy e LMI, Controle \& Automação: Revista da Sociedade Brasileira de Automática 11(1): 37-48.
Teixeira, M. C. M. and Żak, S. H. (1999). Stabilizing controller design for uncertain nonlinear systems using fuzzy models, IEEE Transactions on Fuzzy Systems 15(1): 116-132.

Wang, H. O., Tanaka, K. and Griffin, M. F. (1996). An approach to fuzzy control of nonlinear: stability and design issues, IEEE Transactions on Fuzzy Systems 4(1): 14-23.

Zhang, J. M., Li, R. H. and Zhang, P. A. (2001). Stability analysis and systematic design of fuzzy control systems, Fuzzy Sets and Systems 120(1): 65-72. 